Il gentile invito di Rodrigo Toniol per commentare questo testo mi fa credere che l'intenzione della redazione della Debates do Ner sia quella - come indica il proprio titolo della rivista - di dare luogo a diversi punti di vista, non necessariamente d'accordo con il punto di vista dell'autore. Si tratta ancora di una tematica di riconosciuta importanza che si trova nel centro delle preoccupazioni e dei dibattiti degli studiosi delle pratiche religiose nello spazio pubblico. Pertanto prendo sin dall'inizio un'ottica che favorisce un punto di vista ben diverso da quello presentato nel testo. Questo succede perché porto dalla mia esperienza antropologica una grande diffidenza rispetto ai modelli (sofisticati o no) e credo in una comprensione basata su analisi fortemente appoggiate su categorie sociali native ed anche storiche e situazionali. Conseguentemente, valorizzo qui un approccio forse polemico per dare il via a un dibattito. Vorrei pure evidenziare la qualità analitica del testo che favorisce l'esercizio critico. Questo è ispiratore e i problemi di cui tratta stimolano grande parte dei ricercatori della religione nell'attualità.

Il testo Il senso e il problema del "separatismo moderato".

Per una contribuzione sociologica all'analisi della religione nello spazio pubblico di Luca Diotallevi si propone a discutere la validità e i limiti di certi modelli di separazione tra Chiesa (o Religione) e Stato ${ }^{2}$. Comunque, a differenza di molti, presenta una discussione densa e sofisticata di certe conseguenze teoriche relative all'impiego dei modelli di "separatismo moderato" e "separatismo radicale" per pensare la diversità di casi della relazione tra Chiesa e Stato nell'occidente. Argomenta che la differenza tra il separatismo moderato e il separatismo radicale per qualificare le relazioni della Religione e lo Stato non considerano quello che importa veramente. In altre parole, queste espressioni e il binarismo che le compongono riuniscono nella stessa "casa" classificatoria elementi le quali distinzioni permetterebbero di capire meglio quello che succede

1Vorrei ringraziare Cecília Mariz per la sua precisione sociologica che mi ha aiutato tantissimo.

2L'autore utilizza in modo libero e intercambiabile i termini Religione e Chiesa per quanto riguarda lo Stato (tutti i termini nel singolare e capitalizzati). 
contemporaneamente in questo campo, visto attraverso i modelli di laicità sia radicale che moderata. Insomma, quello che è più importante per Diotallevi è il modo nel quale il modello americano e quello francese - entrambi situati nella stessa chiave di "separatismo radicale" si autorealizzano. Quali sarebbero le configurazioni sociali alla base di questi modelli? Si sarebbero opposti in tutto anche se entrambi possono essere ritenuti vicini nell'usare come criterio la nozione di separatismo, tanto quello radicale quanto il moderato, difende l'autore.

Quindi Luca Diotallevi, ispirato da Luhmann e Stepan (tra altri), continua a discutere come i modelli francese e americano si distinguono - per dimostrare l'inadeguatezza di associarli attraverso la stessa chiave di lettura, quella del separatismo. Come conclusione l'autore suggerisce che attraverso un'analisi del modello di differenziazione sociale è possibile capire meglio quello che succede nell'attualità tra le sfere dello Stato e della Religione. La sua conclusione è pure un modo di affermare come il modello francese sia già esaurito tanto per quanto riguarda la pratica quanto per la teoria - la cosa migliore da fare sarebbe abbandonarlo per analizzare i modelli di differenziazione e le sue conseguenze invece di insistere nei "separatismi". Prevalgono ovunque dei tipi di cooperazione che potrebbero essere meglio compresi attraverso strumenti più adeguati, insiste l'autore.

Religous freedom e laicité sarebbero due matrici concettuali estremamente distinte. Mentre la prima suppone che ci sia nella società americana un processo di differenziazione e di emancipazione delle sfere senza predominare la politica e (ancora meno) lo Stato, la seconda, la laicité francese si basa su una società nella quale lo Stato e la politica dominano le altre sfere. Nella prima, la società americana, l'orizzonte sarebbe l'assenza dello Stato e della Religione come una istanza che fonda la libertà e l'autonomia di tutte le sfere sociali, comprendendo anche quella individuale. L'autore ritiene che nella società statale, quella francese, essendo idealmente omogenea, lo spazio della religione sarebbe sottratto dallo Stato come forza egemonica che attuerebbe contro le differenziazioni delle sfere e le loro emancipazioni. 


\section{COMMENTI SUL “IL SENSO E IL PROBLEMA DEL 'SEPARATISMO MODERATO'. PER UN CONTRIBUTO SOCIOLOGICO ALLA ANALISI DELLA RELIGIONE NELLO SPAZIO PUBBLICO” DI LUCA DIOTALLEVI 3}

Ci rendiamo conto che l'autore si mostra favorevole al modello americano attraverso la valorizzazione degli elementi strutturali di questa società relativi funzionalmente alla nozione di religious freedom.

Alcune questioni

Mi permetto di formulare alcune questioni che in grande misura nascono dalla qualità teorica dei suoi argomenti, anche se non sono sempre formulati chiaramente, secondo il mio parere.

Prima di tutto, ho avuto l'impressione che le distinzioni con le quali l'autore lavora per verificare l'efficacia comparativa dei modelli di "separatismo moderato" e "separatismo radicale" e ancora di "laicità" e "religious freedom" si presentono descritte come "isolati" sociali che operano attraverso le coerenze che gli sarebbero proprie in "canali" esclusivi, diciamo. In questi "canali", l'autore evidenzia cause e conseguenze, evoluzioni e attributi senza che questi siano pensati come minimamente "comunicanti" e inseriti nella vita sociale. Negli scontri storici che hanno stabilito questa opposizione, la differenziazione tra Stato e Chiesa, per esempio in Francia, sarebbe possibile trovare questa nitidezza di modello? Sembra che ci siano attraverso la sua descrizione, entità sociali, fedeli a sé stesse, come Stato e Religione, i cui rapporti non hanno subito nessun intervento al di là delle loro rispettive frontiere e dei propri modi di rapporto. Così, sia la società americana che quella francese possono avere i loro modelli analizzati senza che si faccia riferimento a delle situazioni nelle quali attori molteplici, forze e poteri diversi costituirebbero questi "canali" e interferirebbero in modo permanente "in loro" e "con loro". Sarà possibile produrre questi "isolati" sociali e analizzare i loro effetti senza fare menzione a quello che, diciamo così, gli costituisce nelle complesse arene e trame della vita sociale?

Al secondo posto, mi piacerebbe far menzione all'univocità che, nel testo, assumono le categorie sociali, non soltanto "religione" e "stato" ma pure "democrazia", "autoritarismo", "cooperazione" e "società". Possiamo fare riferimento 
ad alcuni esempi di lavori sullo Stato che scompongono i loro modelli prodotti grossomodo dalla scienza politica ${ }^{3}$. Sarà possibile considerarlo senza discutere quale versione di questa categoria si stia assumendo? Quali sono le forze sociali che ci si trovano e con le quali si collegano? Forse, queste non avrebbero modificato quello che si capiva come Stato? Quali agiscono a nome suo e in quali circostanze? Nella stessa direzione, la nozione di Religione ci sembra veramente instabile nei suoi sensi e impieghi. Basta ricordare il fatto che la nozione di Religione usata qui sembra naturalizzare il fatto che questa non presupponga le stesse pratiche sociali in diverse circostanze e luoghi. Sembra escludere del suo campo alcune cosiddette "sette" da un lato dell'Atlantico (Francia e Germania) e ammetterle dall'altro (Stati Uniti e Brasile, tra altri paesi), ad esempio. Il cosiddetto "insegnamento religioso nelle scuole" in Brasile non è obbligatorio in alcune regioni e tra queste, come il Rio de Janeiro, non include le scuole private, prevalentemente di classe media. In queste ultime, per esempio, il candomblé è spesso previsto nel curriculum con una dimensione estetica nella condizione di folclore

Credo che questi concetti, come sottolineato da alcuni autori, hanno una grande instabilità e il campo sociale al quale si riferiscono si trova sempre più avvolto da nebbie e incertezze. Basta sottolineare gli effetti sui rapporti tra libertà religiosa e sfera politica e giuridica nati nell'11 settembre - le leggi di eccezione, la stigmatizzazione crescente dell'Islam e anche una "contaminazione" della religione nella politica e viceversa che rimane - forse distinta della differenziazione delle sfere presentata dall'autore. Infatti quello che sembra succedere é una dominazione crescente della politica dello stato sulla libertà religiosa nei campioni della libertà religiosa che sono gli Stati Uniti, generandone limitazioni crescenti. Non occorre fare menzione al fatto che la propria geopolitica mondiale oggi sembra essere sottomessa a dei criteri sul ruolo delle chiese, sinagoghe e moschee in diverse parti del mondo.

Non è possibile dall'altra parte, nel caso francese, dissociare la difesa della laicità nei tempi recenti della rivendicazione per una "Europa Cristiana" contro la

3Per esempio, The Anthropology of the State, ed. Veena Das e Deborah Poole, ed. SARAS Press, 2004. 


\section{COMMENTI SUL “IL SENSO E IL PROBLEMA DEL 'SEPARATISMO MODERATO'. PER UN CONTRIBUTO SOCIOLOGICO ALLA ANALISI DELLA RELIGIONE NELLO SPAZIO PUBBLICO” DI LUCA DIOTALLEVI 5}

migrazione africana dalla parte dei settori cattolici e conservatori della società. E ancora, le tensioni sociali relative all'antisemitismo che si trovano tra $\mathrm{i}$ francesi di origine araba, favorevoli - in grande parte - alla creazione di uno stato palestinese - si aggiungono alle controversie che rimangono sulle antiche colonie, che riguardano il multiculturalismo, "l'Europa" e le "razze". Le controversie politiche rispetto all'uso del velo negli spazi pubblici non possono nemmeno essere considerate isolatamente. Si è presentato senza dubbio in un contesto di tensione riguardante la minaccia del "mondo arabo" rappresentato da uno Islam pauroso e onnipresente come nemico, parte della "guerra" contro il "terrorismo" e pure, a un disagio provocato dalle donne di origine africana nel contesto della crescita della migrazione clandestina. Queste "paure" che sembrano non essere distinte nei due lati dell'Atlantico hanno sicuramente le loro specificità.

E dove si trova lo Stato americano? Il controllo sociale e politico sembra non avere i suoi limiti territoriali nello stato-nazione. Il caso di Guantanamo è esemplare come un posto "fuori" del territorio nazionale e sottomesso dallo Stato americano a un regime di eccezione. L'Islam sembra essere presente nella società americana come il fantasma e il contrappunto alla violenza terroristica, associata al radicalismo politico. L'amalgama tra l'islamismo e il terrorismo, che sembra ancora avere una lunga vita in scala globale, ci fa pensare a quanto le nostre categorie analitiche come religione e stato hanno la necessità di essere messe in discussione attraverso gli usi che emergono dalle turbolenze della vita quotidiana.

Traduzido por Ariane Ribeiro Marques

Revisado por Cláudia Mendonça Scheeren 